\title{
THE UNIFIED APPROACH TO SPECTRAL ANALYSIS. II
}

\author{
R. WEDER
}

\begin{abstract}
We apply a new unified method to construct a closed, selfadjoint in $\mathfrak{L}^{2}$, extension of a partial differential operator in all the spaces $\mathfrak{L P}^{P}\left(\mathbf{R}^{n}\right)$, $1<p<\infty$, to a large class of partial differential operators. We obtain very weak conditions in the potentials.
\end{abstract}

Introduction. When one is studying the problem of constructing a closed extension of a partial differential operator in $\mathcal{L}^{p}\left(\mathbf{R}^{n}\right), 1 \leqslant p \leqslant \infty$, intuition tells us (since the basic object is the operator not the $\mathcal{L}^{p}\left(\mathbf{R}^{n}\right)$ space in which it is defined) that for a reasonable class of potentials the operator should have a closed extension (selfadjoint in $\mathfrak{L}^{2}$ if it is formally symmetric) in all the $\mathfrak{L}^{p}$ spaces, $1 \leqslant p \leqslant \infty$. Moreover the spectrum should be essentially the same in all the $\varrho^{p}$ spaces, $1 \leqslant p \leqslant \infty$.

If one tries to prove what intuition tells with the standard methods one is faced with the following problems:

(1) The method of defining the perturbed operator as the operator sum of the unperturbed part plus the perturbation works in $\mathcal{L}^{p}\left(\mathbf{R}^{n}\right), 1 \leqslant p<\infty$. It does not work in $\mathfrak{L}^{\infty}$ as soon as the potentials are allowed to have singularities.

(2) Quadratic form extensions are defined only in $\mathcal{L}^{2}$ and $S$-extensions [2] are defined only in $\mathfrak{L}^{p}, 1<p<\infty$.

(3) Even in the case when one of the standard methods can be applied it provides sufficient conditions for the existence of a closed extension in a given $\mathfrak{L}^{p}$ space, not in all the $\mathfrak{L}^{p}$ spaces at the same time.

In a previous paper [1] we developed a method to fulfill the intuitive program. We applied our method to elliptic partial differential operators. We obtained weaker conditions than the ones obtained with the standard methods.

In this paper we apply our method to the class of partial differential operators introduced by Martin Schechter [2].

Let $P_{0}$ be a constant coefficients partial differential operator of degree $m$ : Let $Q$ be the perturbation: $Q=\sum_{i=1}^{M} q_{i}(x) Q_{i}$. $Q_{i}$ is a constant coefficients partial differential operator of degree $l_{i}<m$.

We consider the class of operators that satisfy the following condition [2]:

Received by the editors February 21, 1978.

AMS (MOS) subject classifications (1970). Primary 35A99, 47A55; Secondary 81A10. 


$$
\begin{aligned}
\frac{D^{\mu} P_{0}(k)}{P_{0}(k)}=O\left(|k|^{-a|\mu|}\right) \quad \text { as }|k| \rightarrow \infty, a \leqslant 1, \\
\frac{Q_{i(k)}}{P_{0}(k)}=O\left(|k|^{-c_{J}}\right) \quad \text { as }|k| \rightarrow \infty, \text { where } c_{J}>a+n-a n .
\end{aligned}
$$

The perturbed operator $P$ is defined as $P=P_{0}+Q$.

We are interested in operators that are formally symmetric. This is not a serious restriction since in most of the applications the operators are formally symmetric. The generalization to the nonsymmetric case is straightforward.

We give our conditions in terms of the quantity [2]:

$$
M_{\alpha}(q)=\sup _{x} \int_{|x-y|<1}|q(x-y)||y|^{\alpha-n} d y, \quad 0<\alpha<n .
$$

$M_{\alpha}$ is the class of functions such that $M_{\alpha}(q)<\infty$.

Our main theorem is:

THEOREM I.1. Let $P$ be a formally symmetric operator satisfying (A). Moreover assume that $M_{\alpha_{i}}(q)<\infty, 1 \leqslant i \leqslant M$, where $0<\alpha_{i}<n-k_{i}, k_{i}$ is the smallest nonnegative integer satisfying $a k_{i}>n-c_{i}$. Finally assume that $\int_{|x-y|<1}|q(y)| d y \rightarrow|x| \rightarrow \infty 0$ uniformly in $x$. Then $P$ defined on $D(P)=\{\phi \in$ $\left.C_{0}^{\infty} \mid P \phi \in \mathfrak{L}^{p}\right\}$ has a closed extension, denoted $H_{p}$, in $\mathfrak{L}^{p}$ for $1 \leqslant p \leqslant \infty . H_{p}$ is densely defined for $1 \leqslant p<\infty$ and $H_{p}^{*}=H_{p^{\prime}}, 1 / p+1 / p^{\prime}=1,1<p<\infty$. In particular $\mathrm{H}_{2}$ is selfadjoint. Moreover the essential spectrum of $\mathrm{H}_{p}$ is given by $\sigma_{e}\left(H_{p}\right)=\left\{P_{0}(k) \mid k \in \mathbf{R}^{n}\right\}, 1 \leqslant p \leqslant \infty$. If $\operatorname{dist}\left(z, \sigma_{e}\left(H_{p}\right)\right)$ is large enough the formal series expansion for the resolvent is convergent in norm, i.e.

$$
\left(z-H_{p}\right)^{-1}=\sum_{n=0}^{\infty} R_{0}(z)\left(Q R_{0}(z)\right)^{n} .
$$

Our method is not only an unified approach, it is also efficient. The conditions of Theorem I.1 are much weaker than the ones obtained by the previously known methods [2]. By the argument developed in [1] it follows that if $P_{0}(k)$ is bounded below the extension obtained with our method coincides, in $\mathfrak{L}^{2}$, with the one obtained by quadratic form methods, i.e. $H_{2}=H$, where $H$ is the quadratic form extension.

It follows as in Theorem II.10 of [1] that the spectrum of $H_{p}, 1 \leqslant p \leqslant \infty$, is real.

The proofs. Let $P_{0}$ be a constant coefficients partial differential operator of degree $m$ :

$$
\begin{aligned}
P_{0} & =\sum_{|\mu|<m} a_{\mu} D_{\mu}, \quad \text { where } D_{u}=\left(D_{1}^{u_{1}}, \ldots, D_{n}^{u_{n}}\right), \\
x & =\left(x_{1}, \ldots, x_{n}\right) \in \mathbf{R}^{n}, \quad D_{k}=-i \partial / \partial x_{k} .
\end{aligned}
$$

We denote

$$
P_{0}(k)=\sum_{|\mu|<m} a_{\mu} k^{\mu}
$$


We assume that $P_{0}(k)$ is real valued. The perturbation, $Q$, is the variable coefficients partial differential operator $Q=\sum_{i=1}^{M} q_{i}(x) Q_{i} \cdot Q_{i}$ is a constant coefficients partial differential operator of degree $l_{i}<m$.

We are interested in operators that are formally symmetric, i.e. such that

$$
(P \phi, \psi)=(\phi, P \psi), \quad \phi, \psi \in D(P) .
$$

As is well known, $P_{0}$ defined in $C_{0}^{\infty}$ (the infinitely differentiable functions of compact support) is closable in $\mathcal{L}^{p}, 1 \leqslant p \leqslant \infty$. We denote by $P_{0, p}$ the closure of $P_{0}$ in $\mathfrak{L}^{p}$, for $p<\infty$. Since the domain of the closure of $P_{0}$ in $\mathfrak{L}^{\infty}$ is too small we define

$$
P_{0, \infty}=P_{0,1}^{*} .
$$

For a linear operator, $H$, in a Banach space, $\mathscr{B}$, we define the resolvent set of $H, \rho(H)$, and the spectrum, $\sigma(H)$, in the usual way. The discrete spectrum $\sigma_{d}(H)$, is the set of isolated eigenvalues of finite multiplicity. We define

Definition II.0. The essential spectrum of a linear operator, $H$, in a Banach space is the complement (in the spectrum) of the discrete spectrum i.e. $\sigma_{e}(H)=\sigma(H) \backslash \sigma_{d}(H)$. We have

$$
\boldsymbol{\sigma}\left(P_{0, p}\right)=\sigma_{e}\left(P_{0, p}\right)=\left\{P_{0}(k) \mid k \in \mathbf{R}^{n}\right\} .
$$

For $p<\infty$ this is a classical result. The case $p=\infty$ follows as in [1].

Proof of TheOREM I.1. The operator $q_{i} Q_{i}$ is $P_{0,1}$-compact [2, Theorem 5.3, p. 92]. Then for $z \notin\left\{P_{0}(k) \mid k \in \mathbf{R}^{n}\right\}$, the operator

$$
Q R_{0}(z)=\sum_{i=1}^{M} q_{i} Q_{i} R_{0}(z)
$$

is compact in $\mathcal{L}^{1}$, where $R_{0}(z)=\left(z-P_{0,1}\right)^{-1}$. Moreover if $\operatorname{dist}\left(z,\left\{P_{0}(k) \mid k \in\right.\right.$ $\left.\left.\mathbf{R}^{n}\right\}\right)$ is large enough $\left\|Q R_{0}(z)\right\|_{\mathcal{L}^{1}, \mathcal{L}^{1}}<1$. Then the formal series expansion of the resolvent of the perturbed operator:

$$
R(z)=\sum_{n=0}^{\infty} R_{0}(z)\left(Q R_{0}(z)\right)^{n}
$$

converges in norm to a bounded operator in $\mathfrak{L}^{1}$ namely:

$$
R_{1}(z)=\sum_{n=0}^{\infty} R_{0}(z)\left(Q R_{0}(z)\right)^{n}=R_{0}(z)\left(1-Q R_{0}(z)\right)^{-1} .
$$

Since $P$ is formally symmetric $R_{0}(z) Q=\left(Q R_{0}(\bar{z})\right)^{*}$. Then $R_{0}(z) Q$ is a contraction on $\mathfrak{L}^{\infty}$. This implies that the formal series expansion defines a bounded operator on $\mathfrak{L}^{\infty}$ :

$$
R_{\infty}(z)=\sum_{n=0}^{\infty}\left(R_{0}(z) Q\right)^{n} R_{0}(z)=\left(1-R_{0}(z) Q\right)^{-1} R_{0}(z) .
$$

By the Riesz-Thorin interpolation theorem the formal series expansion defines a bounded operator in $\mathfrak{L}^{p}, 1 \leqslant p \leqslant \infty$, denoted by $R_{p}(z)$. 
The fact that $R_{p}(z)$ is the resolvent of a closed operator, i.e. $R_{p}(z)=(z-$ $\left.H_{p}\right)^{-1}$, that $H_{p}$ is an extension of $P$ in $\mathfrak{L}^{p}$, and the rest of the theorem follows as in [1]. Q.E.D.

\section{REFERENCES}

1. R. Weder, The unified approach to spectral analysis, Comm. Math. Phys. 60 (1978), 291.

2. M. Schechter, Spectra of partial differential operators, North-Holland, Amsterdam, 1971.

Department of Mathematics, Princeton University, Princeton, New Jersey 08540

Current address: Universidad Nacional Autonoma de Mexico, Apartado Postal 20-72, Mexico 20 D. F., Mexico 